\title{
Le système éducatif américain
}

Laurence Emile-Besse

\section{(2) OpenEdition}

Journals

Édition électronique

URL : https://journals.openedition.org/ries/1760

DOI : $10.4000 /$ ries. 1760

ISSN : 2261-4265

\section{Éditeur}

France Education international

\section{Édition imprimée}

Date de publication : 1 avril 2004

Pagination : 137-144

ISBN : 978-2-85420-560-2

ISSN : 1254-4590

\section{Référence électronique}

Laurence Emile-Besse, "Le système éducatif américain », Revue internationale d'éducation de Sèvres [En ligne], 35 | avril 2004, mis en ligne le 23 novembre 2011, consulté le 05 juillet 2021. URL : http:// journals.openedition.org/ries/1760; DOI : https://doi.org/10.4000/ries.1760

Ce document a été généré automatiquement le 5 juillet 2021.

(c) Tous droits réservés 


\title{
Le système éducatif américain
}

\author{
Laurence Emile-Besse
}

1 Parler du système éducatif américain est une sorte de contradiction dans les termes tant le terme "système» suppose une cohérence et une unité qui s'opposent précisément à ce qu'est l'éducation américaine. Pour ne pas se méprendre, il faut, en préambule rappeler quelques faits.

2 L'immensité du territoire et la structure politique (une fédération) rendent impossible une gestion unique et centralisée de l'école. À titre d'exemple, les écoles sont placées sous la responsabilité des districts, la plus petite unité administrative, qui sont au nombre de seize mille ! Or si cette unité administrative est la plus petite, elle est aussi la plus puissante puisque c'est elle qui décide, par exemple, du programme scolaire, de l'emploi du temps, du salaire, de la formation et du recrutement des enseignants, enjeux qui peuvent donc prendre seize mille formes différentes selon la localisation de l'école.

3 Terre d'immigration, les États-Unis ne cessent d'attirer de nouveaux étudiants (qui viennent à $51 \%$ d'Asie et à $13 \%$ d'Europe) et les écoles américaines ajoutent chaque jour aux cinquante-trois millions d'élèves de nouveaux apprenants, enfants d'immigrés, en situation légale ou non, et qu'il faut intégrer au système scolaire, notamment en leur apprenant l'anglais. Observer les performances scolaires des étudiants américains aux tests internationaux sans cette donnée, c'est oublier que la population scolaire américaine est extrêmement contrastée et changeante, les hispanophones étant, par exemple, en passe de devenir la première «minorité majoritaire » devant les Noirs. Signe de cette difficile intégration, le pourcentage d'abandon en cours d'études : $7 \%$ pour les Blancs, $13 \%$ pour les Noirs et encore $28 \%$ pour les Hispaniques. Conséquence positive toutefois, les écoles américaines changent, se diversifient et créent de nouveaux programmes de types alternatifs (magnet schools, charters schools) destinés à aider les plus défavorisés.

4 Immensité, décentralisation, contrastes : ces trois termes permettent de comprendre les difficultés et les réussites de l'école américaine. Depuis près d'une vingtaine d'années, l'État fédéral a pris conscience de ces problèmes. En contradiction avec la Constitution, il cherche à intervenir davantage dans les politiques éducatives des États. 


\section{Une politique éducative décidée localement}

En matière d'éducation, la répartition des pouvoirs se fait à trois niveaux.

\section{Niveau fédéral}

Le Department of Education à la tête duquel se trouve le Secretary of Education ne contribue qu'à hauteur de $7 \%$ au budget des établissements publics, les $93 \%$ restants relevant du local. Il a pour fonction d'assurer l'égalité d'accès de chaque citoyen au système éducatif du pays, d'améliorer l'éducation par des programmes spécifiques, de centraliser les données, statistiques et recherches sur le thème de l'éducation pour ensuite en assurer la diffusion auprès des États. Toutes les autres questions éducatives relèvent de la structure locale, État d'abord, puis district.

\section{Niveau des États}

7 À la tête de chaque État, on retrouve un Departement of Education dont les fonctions sont locales. Il est présidé par le commissioner of education associé à un board of education dont les membres sont soit nommés par le gouverneur de l'État soit élus par le peuple ou ses représentants. L'État doit mettre en œuvre les programmes spécifiques décidés au niveau fédéral mais c'est lui définit l'ensemble de la politique scolaire à mener en termes de choix des programmes, calendrier, habilitation des enseignants, administration des tests (dont la conception est toutefois confiée à des organismes privés).

\section{Niveau des districts}

8 Dans les faits, le pouvoir des États est en partie délégué aux seize mille school districts administrés par des conseils locaux, les schools boards. À la tête du school district se trouve le superintendent, homologue local du commissioner of education. Le district relaie la politique de l'État et a aussi pour rôle de recruter les enseignants (définir leur niveau de qualification, leurs certifications, assurer leur formation) et de fixer leurs salaires, d'établir les programmes scolaires, les méthodes pédagogiques et l'emploi du temps des établissements.

\section{Une tendance récente : I'implication de plus en plus forte du gouvernement fédéral}

Le Department of Education fut créé en 1867 avec mission de rassembler des informations sur les écoles et le système d'enseignement afin d'améliorer l'efficacité du système scolaire. Si le pouvoir fédéral en matière d'éducation est restreint, il tend depuis la fin de la seconde Guerre mondiale à s'affirmer et à se développer.

En 1890, le Department devint Office of Education et vit ses responsabilités accrues en devenant le principal pourvoyeur de bourses pour les étudiants. Autre fonction nouvelle: l'enseignement professionnel qui, en 1917 puis 1946, devint une des 
nouvelles priorités de l'office of Education. Notons à cet égard que les deux conflits mondiaux accrurent le pouvoir fédéral en matière d'éducation : le retour d'anciens combattants souvent jeunes et ayant dû interrompre leurs études obligea le gouvernement fédéral à créer ou à développer de nouvelles structures pour le retour des étudiants partis à la guerre. Ainsi, en 1944, le GI Bill permit de fournir une aide universitaire à près huit millions d'anciens combattants.

11 La guerre froide constitua la deuxième étape de l'affirmation du pouvoir fédéral en matière d'éducation : le lancement du Spoutnik en 1957 est, aujourd'hui encore, vécu comme le choc national, la prise de conscience majeure des déficiences du système éducatif américain. L'année suivante, le Congrès votait le National Defense Education Act qui avait pour fonction d'améliorer la formation des chercheurs et étudiants en sciences, d'augmenter l'aide financière aux étudiants et, enfin, de développer et d'améliorer l'enseignement des langues étrangères dans les écoles primaires et secondaires.

12 À l'intérieur des frontières mêmes, c'est le mouvement des Civils Rights, dans les années soixante à soixante-dix, qui renforça le rôle de l'office of Education. Sous la pression des mouvements de défense des droits des Noirs, l'office of Education se vit chargé d'assurer l'égalité d'accès aux études: le Elementary and Secondary Education Act (ESEA), voté en 1965, visait à rétablir l'égalité des chances (equal opportunity) pour les catégories les plus défavorisées, le Rehabilitation Act de 1973 interdisant quant à lui toute discrimination fondée sur la couleur, le sexe ou le handicap. Une des mesures gouvernementales les plus marquantes de cette époque est le programme Head Start, série de mesures tant sanitaires qu'éducatives visant à aider les enfants défavorisés de trois à cinq ans dans un pays ou les maternelles étaient encore embryonnaires.

En 1980, l'Office of Education acquiert une dimension ministérielle en devenant Department of Education. Au début des années quatre-vingts, le gouvernement Reagan commande un rapport sur l'état des écoles américaines : ce sera $A$ nation at risk, un rapport très alarmiste sur les défaillances du système éducatif américain et la faiblesse des résultats de ses élèves. À l'époque déjà, $A$ nation at risk est considéré comme très orienté, les résultats sujets à caution et cherchant à provoquer un sursaut facilitant à terme la "libéralisation du marché éducatif». Exemple emblématique de cette tendance, les vouchers, chèques éducation donnés par le gouvernement fédéral aux parents désirant retirer leur enfant d'une école publique jugée défaillante pour l'inscrire dans une école privée (religieuse ou non). Pour leurs partisans, les vouchers permettent de rétablir l'égalité de chances pour tous les élèves et donc, implicitement, de lutter contre une ségrégation raciale toujours très présente dans les écoles. Mais ils permettent aussi au gouvernement républicain de s'attirer les faveurs de l'électorat noir, traditionnellement démocrate. Enfin, ils posent très crûment la question cruciale du choix de l'école, puisque les parents, devenus consommateurs d'écoles, transforment ainsi l'éducation en marché. Ronald Reagan lui-même déplorait qu'au pays du libre échange et de la loi anti-trust, l'éducation soit demeurée le dernier des monopoles. En ce sens, les vouchers ont aussi comme fonction avouée d'établir une concurrence entre les établissements scolaires, obligés de s'améliorer pour pouvoir rivaliser. 


\section{Le programme No child left behind}

L'implication la plus récente du gouvernement fédéral dans la politique éducative des États est le lancement, en 2001, du programme No child left behind du gouvernement Bush, présenté comme la plus grande réforme éducative des trente dernières années. Il se fixe comme ambition de donner à tous les élèves américains d'ici 2014 un niveau satisfaisant en lecture et en mathématiques. À plus court terme, l'objectif est aussi que chaque classe ait « un enseignant hautement qualifié ».

Le programme s'organise selon les principes suivants :

- responsabilité : l'État, le district et l'établissement scolaires sont responsables des résultats des élèves ; pour ce faire, des standards seront établis par États et les élèves seront de plus en plus souvent testés en mathématiques et en lecture; si une augmentation du niveau des élèves ne se produit pas, État, district et école en seront tenus responsables ;

- flexibilité : les États pourront utiliser l'argent fédéral de façon plus souple et en réduisant les procédures administratives; toutefois, les crédits fédéraux ne sont évidemment versés qu'aux États qui acceptent de mettre en place le programme, obligation qui, dans les faits, constitue une entrave à la liberté en matière de politique éducative dont les États jouissaient jusqu'alors ;

- concentration des dépenses sur «des méthodes d'enseignement qui ont démontré leur efficacité » : cette partie comporte une aide fédérale de quatre milliards pour améliorer la formation des enseignants ;

- un choix plus large pour les parents : les familles pourront bénéficier d'aides fédérales leur permettant de changer leur enfant d'école si celle-ci est jugée déficiente; malgré la ressemblance avec le système des vouchers, il n'est pas précisé toutefois si l'argent fédéral permettra le transfert d'un élève d'une école publique à un établissement privé confessionnel ; une précision stipule que s'il ne sert pas à changer l'enfant d'école, le chèque éducation pourra être utilisé pour des cours de rattrapage assurés par tous types d'organisation, y compris religieuses ; c'est donc sous une forme détournée, renouer avec le principe le plus controversé des vouchers qui, en utilisant des fonds publics pour des écoles privées, notamment religieuses, ne respectait pas la séparation de l'Église et de l'État.

\section{Un système encore très inégalitaire}

\section{Le contraste des chiffres}

Chercher à comprendre les États-Unis en trouvant une raison à toute chose est un leurre. Mieux vaut, dans ce pays immense où les extrêmes se rejoignent, accepter la règle du contraste, en indiquant notamment quelques chiffres : entre 2000 et 2003, le budget fédéral pour l'éducation est passé de 38,5 à 63 milliards de dollars, part qui représente toutefois moins de $7 \%$ du budget de l'État. Dans le même temps, le coût de l'entrée dans les universités publiques a augmenté de $28 \%$ en deux ans et nombre d'écoles situés dans les quartiers les plus défavorisés n'ont même pas le matériel nécessaire pour travailler. C'est l'un des effets de la décentralisation du système: le revenu des écoles vient de l'impôt foncier. Selon qu'elle est située dans un quartier aisé ou non, l'école aura ou non les moyens non seulement de se procurer le matériel nécessaire mais aussi d'attirer ou non les meilleurs enseignants. Rappelons en effet que les sept millions d'enseignants n'étant pas payés par l'État fédéral, les disparités en 
matière de salaires sont très fortes. L'écart peut ainsi aller de 31383 dollars dans le Dakota du sud à 54348 dollars en Californie. Le salaire moyen pour les enseignants du primaire et du secondaire était en 1999 de 30719 dollars par an. Ce chiffre est d'ailleurs en augmentation au vu des difficultés qu'ont les établissements à trouver des enseignants.

\section{Des inégalités sociales et ethniques encore très marquées}

L'année 2004 a vu la commémoration de l'arrêt Brown vs Board of Education, par lequel la Cour Suprême interdisait la ségrégation raciale dans toutes les écoles américaines. En janvier dernier, un rapport paru à cette occasion constate que, cinquante ans après, la situation n'a guère changé. Dans certains endroits, elle serait même pire qu'avant 1954. C'est cette difficulté à faire disparaitre la ségrégation dans les écoles qui explique la création du busing, des magnet et des charter schools. Les résultats des élèves sont d'ailleurs scrupuleusement analysés en fonction de leur appartenance ethnique : ainsi, les compétences en lecture, notées sur une échelle de 0 à 500 sont en moyenne de 294 pour les Blancs, 270 pour les Hispaniques, 263 pour les Noirs. Les résultats obtenus dans le privé sont sensiblement meilleurs : 307 pour les élèves du privé contre 285 pour ceux du public; en mathématiques : 315 pour les Blancs, 293 pour les Hispaniques et 283 pour les Noirs. Là encore, le secteur privé obtient de meilleurs scores : 321 en moyenne contre 307 pour le public.

Contraste encore, le corps enseignant lui-même est composé à 73 \% de femmes (à 87 \% blanches) dans le public et $92 \%$ dans le privé. À l'image de leur salaire, le niveau de formation des enseignants diffère beaucoup d'une école à l'autre. En effet, l'absence de concours ou d'examen de niveau national et la liberté donnée à chaque État de définir lui-même son mode de certification crée une très forte hétérogénéité des qualifications des enseignants américains. Le problème est d'autant plus sensible que, dans les dix prochaines années, les États-Unis devront remplacer deux millions d'enseignants partant à la retraite. Déjà, des accords ont été passés avec certains pays (France, Autriche, Espagne) pour faire venir sur le territoire américain des enseignants européens.

\section{Contre toutes les ségrégations : des écoles pour élèves gays et lesbiens}

L'idée peut surprendre ailleurs que dans un pays multicommunautariste. En 2001 a ouvert officiellement à New York la Harvey Milk School, du nom d'un militant de la cause homosexuelle assassiné dans les années soixante-dix. Depuis plusieurs années déjà, des articles traitaient des discriminations souvent très violentes rencontrées à l'école par les jeunes homosexuels. Des campagnes de sensibilisation avaient été menées localement mais la création de l'école de New York est une première, très controversée d'ailleurs non seulement par les partis conservateurs mais aussi par ceux qui voient à terme se profiler une école multicommunautariste au point de ne plus être intégratrice. 


\section{Face aux problèmes scolaires, de nouvelles écoles et de nouveaux programmes}

Inquiets pour leurs écoles et leurs enfants, les Américains ont, depuis une quinzaine d'années, créé de nouveaux cursus destinés notamment à rétablir une certain équilibre entre écoles dans un pays où la situation des établissements est pour le moins contrastée.

21 Signe de l'implication grandissante du fédéral dans la politique éducative des États, ces programmes novateurs sont des initiatives locales qui, lorsqu'elles ont démontré leur efficacité, sont alors incluses dans l'ensemble des mesures fédérales.

\section{L'éducation préscolaire}

Depuis près de trente ans, les États-Unis accordent de plus en plus d'importance à l'enseignement préscolaire : de 1970 à 1999, on est ainsi passé, pour les enfants âgés de trois et quatre ans, de 20,5 millions à 54,2 millions d'enfants scolarisés. Entre 1989 et 1999 , le nombre d'enfants de trois à cinq ans scolarisés dans une institution préscolaire a augmenté de $30 \%$.

Dans la plupart des États, les enfants ne peuvent être scolarisés qu'à partir de cinq ans. Ils entrent alors en pre-kindergarten qui les prépare au first grade à l'instar d'une dernière année de maternelle (apprentissage de la lecture, de l'écriture, des mathématiques). Les parents qui souhaitent scolariser leur enfant avant l'âge de cinq ans doivent choisir le système privé (à raison de mille dollars par mois en moyenne). Certains États proposent cependant des programmes de scolarisation précoce dans le système public. C'est le cas par exemple de la Georgie, où près de $40 \%$ des enfants de quatre ans sont scolarisés. D'autres États, comme la Californie, suivent cette voie en envoyant notamment des experts en France ou en Italie pour étudier le fonctionnement des écoles maternelles. L'éducation préscolaire pour tous est considérée comme un des moyens d'améliorer le système public américain en permettant cependant aux enfants de milieux les plus défavorisés d'être scolarisés dès le plus jeune âge.

\section{Des établissements alternatifs}

\section{Les magnet schools}

Qu'il s'agisse de magnet schools, de charters ou d'immersion schools, toutes apparaissent dans les années soixante-dix au moment du mouvement des civils rights qui rend plus aiguë la question d'un enseignement de qualité pour tous. La prise de conscience qui a suivi le lancement du Spoutnik a eu lieu peu de temps auparavant. C'est donc à un problème intérieur (lutter contre la discrimination raciale) et à un problème extérieur (concurrence soviétique) que les États-Unis doivent faire face. La solution du busing (assurer la mixité sociale en transportant les enfants blancs des quartiers riches vers les écoles des quartiers noirs) ayant échoué, un autre type de solution est envisagé avec le même objectif : créer dans les écoles une diversité sociale permettant de désenclaver les écoles noires ghettoïsées. C'est dans ce but que sont créées les magnet schools, ces programmes d'excellence dans des écoles défavorisées permettant, à l'instar du busing, 
d'attirer les meilleurs élèves dans des écoles des quartiers pauvres. Le français figurant parmi les matières considérées comme difficiles voire élitistes, il n'est pas rare de trouver des programmes de français dans des magnet schools ou que les écoles d'immersion soient des magnet schools.

\section{Les écoles d'immersion} continuer à parler français. Dans cette perspective, ils ont créé des écoles où les enfants se trouvent, dès l'âge de cinq ans, entièrement immergés dans une langue qui n'est pas leur langue maternelle. Exportée aux États-Unis, la formule a rencontré un certain succès. Elle permettait en effet de régler à la fois le problème interne de la discrimination et celui externe de la concurrence avec les Soviétiques (accent mis sur l'enseignement des langues étrangères). Les programmes d'immersion peuvent commencer au niveau élémentaire (immersion précoce) ou secondaire (middle school, immersion tardive) selon un programme partiel (demi-journée en anglais et demijournée en français) ou total. Généralement, la distinction se fait plutôt selon les matières : matières fondamentales dans la langue d'immersion et dessin ou musique en anglais. Aujourd'hui, les programmes d'immersion existent dans plus de dix-sept langues dont l'espagnol, le français, le japonais, le mandarin ou l'inupiaq. En 2001, douze mille élèves américains suivaient un programme d'immersion en français.

\section{Les charter schools}

Ce sont des écoles à financement public mais à gestion privée sous contrat (charter) : en échange d'une plus grande autonomie de fonctionnement (l'école n'est pas placée sous l'autorité du superintendant et peut déterminer seule son programme), la charter school s'engage à atteindre certains objectifs qui, s'ils ne sont pas atteints au bout de trois ou cinq ans, peuvent entraîner la révocation du contrat et la fermeture de l'école. Ce fut par exemple le cas à Washington DC en 2001 où, après une inspection, trois des dix-sept écoles de la ville ont été fermées : le taux d'absentéisme y était de $100 \%$ et les élèves n'avaient pas de manuels.

Les charter schools ont connu sous le gouvernement Clinton une très forte croissance ${ }^{1}$. La vogue des charters schools a plusieurs causes : les écoles y sont plus petites (moins de deux cents élèves), elles assurent donc un meilleur encadrement des élèves en difficulté, la discipline y est plus stricte, les parents plus impliqués et elles semblent favoriser une meilleure mixité sociale. L'obligation de résultats rend l'école « redevable » (accountable) - terme clef du système éducatif américain qui espère ainsi responsabiliser l'école et l'améliorer. Enfin les charter schools sont encore le dernier rempart contre le système des vouchers qui, parce qu'il est plus radical, menace davantage la séparation entre l'Église et l'État.

Si ce type d'école est particulièrement prisé actuellement, il n'est pourtant pas exempt de problèmes: l'AFT (l'American Federation of Teachers) recommande vivement aux charter schools d'adopter des critères d'exigence plus élevés et de n'embaucher que des enseignants ayant obtenu leur certification... 


\section{Le homeschooling}

29 préserver l'éducation religieuse de leurs enfants, le homeschooling a connu depuis dix ans une ascension foudroyante en triplant le nombre de ses élèves. L'éducation à la maison est, aux yeux de ses défenseurs, un remède aux déficiences du système éducatif et notamment au manque de moyens des écoles publiques. Le développement des nouvelles technologies a donné un soutien inattendu aux homeschoolers aujourd'hui parfaitement organisés en réseau. Cet enseignement à la carte a tout pour séduire une société multicommunitariste soucieuse de préserver les valeurs de son groupe d'appartenance d'autant que les Américains ont une très mauvaise image de leurs écoles. Les enfants n'étant généralement pas scolarisés avant l'âge de cinq ans, le coût des frais de garde incite nombre de femmes américaines à cesser de travailler, tradition qui trouve dans la pratique du homeschooling un prolongement logique.

\section{Des réformes possibles?}

Les deux principaux syndicats d'enseignants américains l'American Federation of Teachers et la National Education Association recommandent les mesures suivantes pour tenter de pallier les déficiences du système éducatif américain :

- rendre l'éducation préscolaire accessible à tous sur le modèle du système français des maternelles;

- réduire les effectifs dans les classes ;

- améliorer la formation des enseignants afin qu'ils soient tous qualifiés dans la matière qu'ils enseignent, en leur adjoignant par exemple des tuteurs;

- rendre la profession plus attractive en augmentant les salaires et en améliorant les conditions de travail dans les zones les plus difficiles;

- développer une politique de soutien aux enfants en échec : cours de soutien, accueil pendant l'été, extension du programme Early Start à davantage d'enfants défavorisés. 
Organisation du système éducatif américain

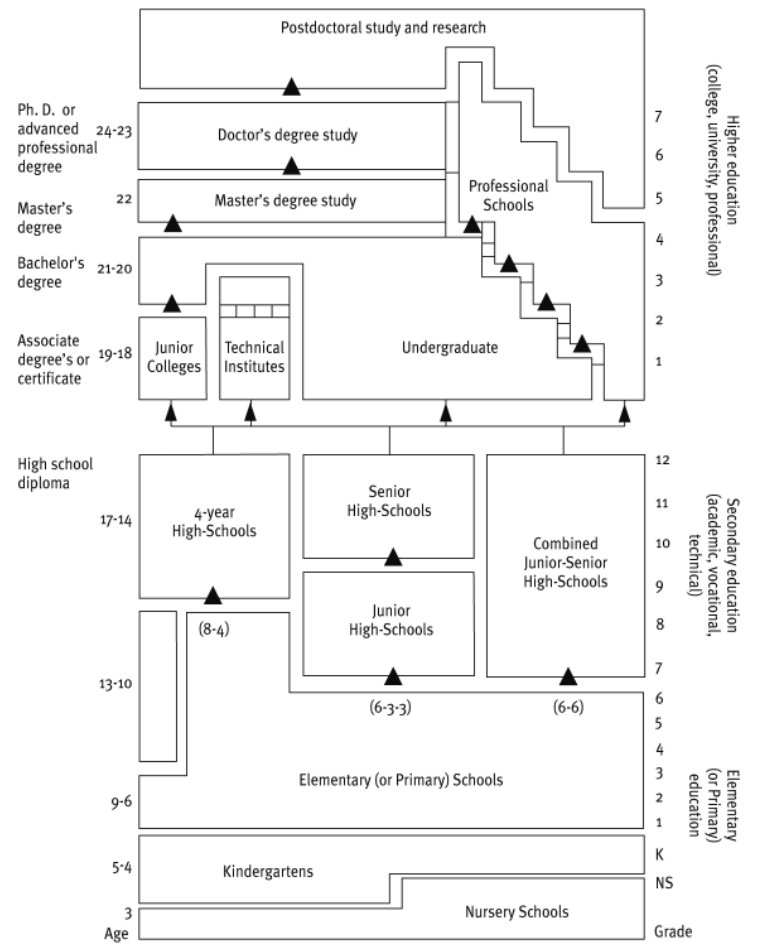

Source: U.S. Office of Education, Digest of Educational Statistics. i972(OE 73-19104). Washington, D.C. Département of Health, Education, and Welfare, p. 33

\section{NOTES}

1. 800 ont été créées entre 1991 et 1998 . Elles sont 2150 sur tout le territoire américain.

\section{INDEX}

Mots-clés : système éducatif

Index géographique : États-Unis 
AUTEUR

\section{LAURENCE EMILE-BESSE}

Ambassade de France à Washington 